\title{
Emission of collimated THz pulses from photo-excited semiconductors
}

\author{
Michael B Johnston ${ }^{1}$, Annette Dowd ${ }^{2,5}$, Robert Driver ${ }^{2}$, \\ Edmund H Linfield ${ }^{2}$, A Giles Davies ${ }^{3}$ and David M Whittaker ${ }^{4}$ \\ ${ }^{1}$ Department of Physics, University of Oxford, Clarendon Laboratory, Parks Road, \\ Oxford OX1 4JF, UK \\ ${ }^{2}$ Department of Physics, University of Cambridge, Cavendish Laboratory, Madingley Road, \\ Cambridge CB3 0HE, UK \\ ${ }^{3}$ School of Electronic and Electrical Engineering, University of Leeds, Leeds LS2 9JT, UK \\ ${ }^{4}$ Department of Physics and Astronomy, University of Sheffield, Sheffield S3 7RH, UK \\ E-mail: M.Johnston1@physics.ox.ac.uk
}

Received 28 July 2003

Published 15 March 2004

Online at stacks.iop.org/SST/19/S449 (DOI: 10.1088/0268-1242/19/4/147)

\begin{abstract}
It is shown experimentally that surface-field terahertz (THz) emitters can produce well-collimated beams of $\mathrm{THz}$ radiation, making them useful devices for time-domain spectroscopy applications. Simulations of the carrier-dynamics are used to explain the mechanism of THz generation in InAs and GaAs, and it is shown that inter-valley scattering of electrons must be considered in order to fully describe $\mathrm{THz}$ emission from InAs.
\end{abstract}

(Some figures in this article are in colour only in the electronic version)

\section{Introduction}

There is currently much interest in exploiting radiation from the far-infrared, or terahertz ( $\mathrm{THz}$ ), region of the electromagnetic spectrum. Coherent pulses of THz radiation, which may be as short as a fraction of an optical cycle, are becoming effective tools for spectroscopy [1-3] and imaging [4] in many areas of science and industry.

Coherent $\mathrm{THz}$ pulses may be generated by optical rectification [5], photoconductive switches [6] and surfacefield emitters [7]. This paper concentrates on surface-field emitters, in particular GaAs and InAs based devices. These devices are simple to align and can be integrated with a wide range of laser systems from low-power Ti:sapphire oscillators to high-power laser amplifiers. We show that surface-field $\mathrm{THz}$ emitters produce very well-collimated pulses of $\mathrm{THz}$ radiation. Furthermore we use a Monte Carlo simulation of the carrier dynamics to explain the differing mechanisms of surface-field $\mathrm{THz}$ generation in InAs and GaAs.

\footnotetext{
5 Present address: Department of Applied Physics, University of Technology Sydney, PO Box 123, Broadway, NSW 2007, Australia.
}

\section{Collimation}

A surface-field $\mathrm{THz}$ emitter typically consists of an unprocessed semiconductor wafer onto which short nearinfrared laser pulses are incident. The laser pulse creates a population of electrons and holes which separate on a picosecond timescale. Thus, a transient dipole develops with its axis perpendicular to the semiconductor surface. The halfcycle oscillation of this vertical $\mathrm{THz}$ dipole typically radiates a picosecond duration pulse containing frequency components between $100 \mathrm{GHz}$ and several THz.

If the illuminating laser beam-waist is much larger than the wavelength of the $\mathrm{THz}$ radiation then the emitted $\mathrm{THz}$ wave front will follow the wave front of the incident laser pulse. This effect can be understood in terms of a simple Huygens picture: the wave front of the laser pulse generates a coherent ensemble of transient dipoles, which radiate $\mathrm{THz}$ transients inphase with the incoming wave front. Therefore manipulation of the incident laser beam can be used to form well-collimated $\mathrm{THz}$ beams; focus a $\mathrm{THz}$ beam at a particular position; or to form an image. It is the ability to create a coherent ensemble of dipoles over a large uniform device that makes surface-field THz emitters attractive for many applications.

We performed a set of experiments to demonstrate this effect. A wafer of (100) InAs (residual n-type carrier 

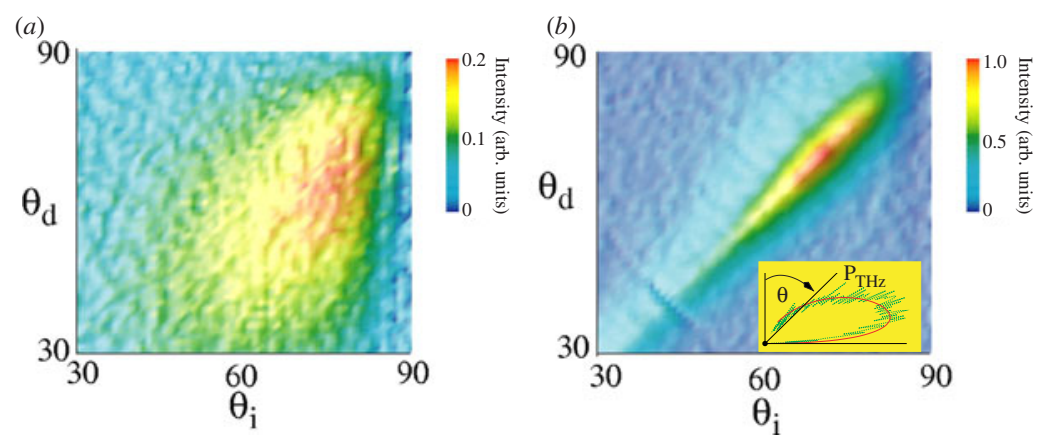

Figure 1. Emission of THz radiation from an InAs surface excited with Gaussian beams from a Ti:sapphire laser, $(a) \sigma=85 \mu \mathrm{m}$, (b) $\sigma=800 \mu \mathrm{m}$. The detector was a Golay-cell bolometer with diamond windows. Reflected and scattered laser radiation was removed using a cellulose nitrate film and a high resistivity silicon filter. The effectiveness of the filter in just passing only the pulsed THz radiation was confirmed by the absence of a bolometer signal when the Ti:sapphire laser was not mode-locked. In addition, a null signal was recorded when the InAs emitter was replaced with a mirror, indicating the system was not influenced by reflected laser light. Inset: Polar plot of emitted THz power in $(b)$ where $\theta_{i}=\theta_{d}=\theta$.

concentration of $3.0 \times 10^{16} \mathrm{~cm}^{-3}$ ) was mounted on a motorized rotation stage with its surface coincident with the axis of the stage. A second rotation stage was used to rotate a $\mathrm{THz}$ detector (Golay-cell bolometer) in a circle around the sample's rotation axis. The overall resolution of the system was $2^{\circ}$. A fixed beam of $\approx 100 \mathrm{fs}$ pulses from a mode-locked Ti:sapphire laser was incident at the sample on the rotation axis. In this way it was possible to map the $\mathrm{THz}$ power emitted from the device as a function of the angle of incidence of the laser beam, $\theta_{i}$, and the angle of the THz detector, $\theta_{d}$, taken with respect to the surface normal of the emitter.

The results of these measurement are shown in figure 1, where the scale represents $\mathrm{THz}$ power as a function of $\theta_{i}$ and $\theta_{d}$. In figure $1(a)$ the incoming laser was focused to a Gaussian beam-waist with $\sigma=80 \mu \mathrm{m}$. In this case the spot size is comparable with the $\mathrm{THz}$ wavelength and the emission pattern (THz power versus $\theta_{d}$ ) starts to approach that of a point dipole for a range of $\theta_{i}$. However, when the laser beam-waist was set to $\sigma=800 \mu \mathrm{m}$ (figure $1(b)$ ) it can be seen that the THz power is maximized when $\theta_{i}=\theta_{d}$. The inset of figure 1 shows the calculated [8] radiation pattern (red/dark grey line) of a point vertical dipole within the sample compared with the measured THz power along the line $\theta_{i}=\theta_{d}=\theta$ (green/light grey line). The collimated experiment and the theoretical point dipole radiation pattern agree remarkably well.

These results demonstrate that, within the diffraction limit, it is possible to reproduce a near-infrared laser pulse's wave front in the wave front of a $\mathrm{THz}$ pulse. In addition, by measuring radiation patterns using a collimated laser in a $\theta_{i}=\theta_{d}=\theta$ geometry it is possible to reproduce the radiation pattern of a point dipole. This is important, as it means that calculations need only consider the simple case of a point dipole. In addition, it shows that the THz dipole's orientation is important irrespective of collimation effects.

\section{Mechanisms of 'surface-field' THz generation}

The two main mechanisms of surface-field $\mathrm{THz}$ generation are an inbuilt surface electric field effect [5] where carriers are driven by an electrostatic force and a photo-Dember effect [9] where the higher mobility of electrons, combined with the asymmetry created by the semiconductor's surface, leads to

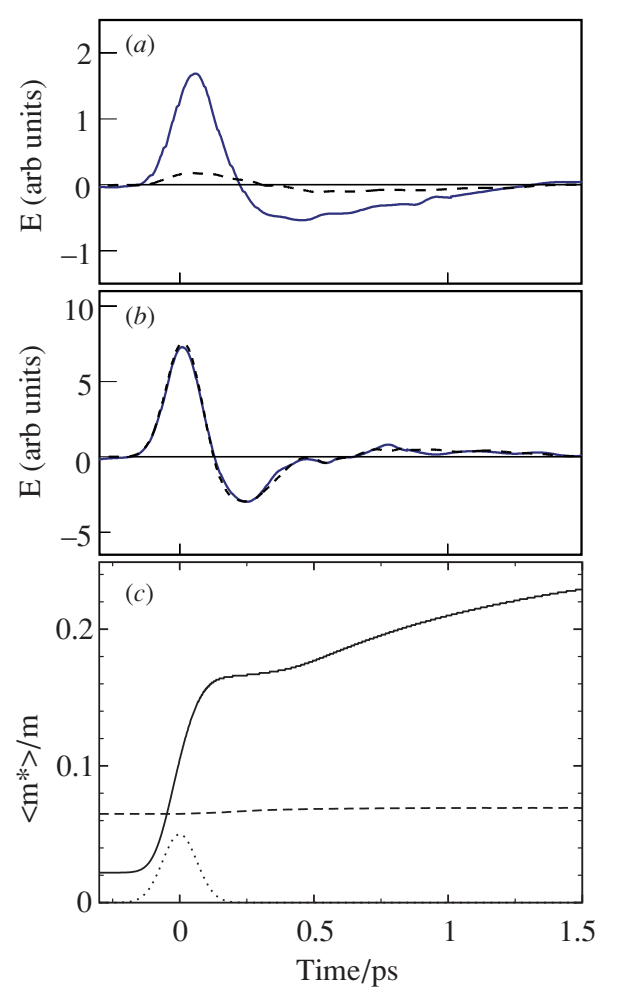

Figure 2. Results of a carrier dynamics simulation of n-type InAs and GaAs during photo-excitation by a typical Ti:sapphire laser pulse (see [10] for detailed parameters of the simulation). (a) and (b) show the electric field of the $\mathrm{THz}$ transients as a function of time for GaAs and InAs, respectively. In both panels the solid line shows a full simulation, while the dashed line shows results when the built-in surface electric field is ignored. (c) shows the average effective mass of electrons in the GaAs (dashed line) and InAs (solid line) simulations as a function of time. The dotted line represents the intensity profile of the near-infrared laser pulse.

a charge separation. We developed a semi-classical carrier dynamics simulation in order to model the $\mathrm{THz}$ emission processes in a variety of semiconductors [8, 10]. The simulation considered the dynamics of both extrinsic and hotphoto-injected charge carriers. Figure 2 shows simulations of $\mathrm{THz}$ generation in n-type GaAs and InAs excited with 
150 fs-pulses from a mode-locked Ti:sapphire laser. To distinguish between the two generation mechanisms simulations were run with and without an inbuilt surface electric field. The GaAs simulations presented in figure 2(a) show that $\mathrm{THz}$ emission is much stronger if the inbuilt surface electric field is included (solid line) compared with the case where it is absent (dashed line). In contrast, the data from the InAs simulations in figure $2(b)$ are almost indistinguishable, showing that the inbuilt surface electric field has little effect on $\mathrm{THz}$ emission from InAs. Thus GaAs is primarily a inbuilt surface-field type emitter while n-type InAs is primarily a photo-Dember type emitter.

These results can be explained by the presence of an accumulation field in n-InAs compared with a depletion field in GaAs; furthermore, the density and temperature of the electron hole plasma in the InAs sample, under the same excitation conditions, are both much higher than in the GaAs sample, thus favouring the photo-Dember mechanism. It is well known that InAs is a more efficient emitter than GaAs, and this phenomenon is reproduced in the simulation (compare the E-axes in figures $2(a)$ and $(b)$ ). However, it is important to note that this is not related to the much lighter $\Gamma$-minimum electron effective mass in InAs compared to that in GaAs, but rather a result of the differences in the carrier densities and generation mechanisms between the two emitters.

Figure 2(c) shows the average effective mass of electrons as a function of time during the simulation. The average effective mass includes all electrons within the volume of the simulation. In both simulations, before the laser pulse arrives the electron effective masses correspond just to the $\Gamma$ minimum values. However, as the laser pulse arrives it can be seen that the average effective mass rises rapidly in the case of InAs, but not for GaAs. Since InAs is a narrow band gap material, the carriers are injected high in the conduction band and readily scatter into the L-valleys, thereby increasing the average effective mass of the electron population. It is interesting to note that the effective mass of the electron population continues to rise, even after the injection has finished. This is a result of energy transfer between the hot photo-injected electrons and the extrinsic donated electrons.

The increased effective mass of the electron population in InAs compared with GaAs can be observed when a magnetic field is applied to these emitters. A magnetic field acts to rotate the vertical THz-emitting dipole, and is observed as a change in the coupling of $\mathrm{THz}$ radiation out of the semiconductor (see [8] for a detailed explanation). Experiments [11-13] show that the magnetic-field induced rotation of the $\mathrm{THz}$ dipole in InAs is much less than in GaAs, which is consistent with our simulation results [10]. The reason for this is that in InAs electrons are scattered relatively quickly to the lower mobility side valleys, which reduces the dipole rotation.
It should be noted that the efficiency of surface-field emitters may be improved by more than an order of magnitude by applying a magnetic field and hence re-orientating the $\mathrm{THz}$ dipole so that a greater fraction of the radiation is coupled into free space [8]. A similar improvement in device efficiency can be achieved, without the need for a magnetic field, by careful design of the emitter geometry. This has led to the development of a compact, efficient prism based $\mathrm{THz}$ source [14].

\section{Conclusion}

For many applications it is useful to create collimated beams of $\mathrm{THz}$ radiation. We have measured collimated $\mathrm{THz}$ emission from InAs as a function of the laser beam-waist. A simulation of carrier dynamics was used to show that when excited by a mode-locked Ti:sapphire laser, InAs is a photo-Dember type emitter, while $\mathrm{THz}$ emission from GaAs is primarily driven by an inbuilt surface electric field.

\section{Acknowledgments}

The authors wish to acknowledge the support of the EPSRC, The Royal Society and Toshiba Research Europe Ltd.

\section{References}

[1] Han P Y, Cho G C and Zhang X C 2000 Opt. Lett. 25242

[2] Huber R, Tauser F, Brodschelm A, Bichler M, Abstreiter G and Leitenstorfer A 2001 Nature $\mathbf{4 1 4} 286$

[3] Johnston M B, Herz L M, Khan A L T, Koehler A, Davies A G and Linfield E H 2003 Chem. Phys. Lett. 377256

[4] Ferguson B and Zhang X-C 2002 Nature Mater. 126

[5] Zhang X C and Auston D H 1992 J. Appl. Phys. 71326

[6] Grischkowsky D, Keiding S, Vanexter M and Fattinger C 1990 J. Opt. Soc. Am. B 72006

[7] Dekorsy T, Pfeifer T, Kütt W and Kurz H 1993 Phys. Rev. B 473842

[8] Johnston M B, Whittaker D M, Corchia A, Davies A G and Linfield E H 2002 J. Appl. Phys. 912104

[9] Dember H 1931 Phys. Z. 32554

[10] Johnston M B, Whittaker D M, Corchia A, Davies A G and Linfield E H 2002 Phys. Rev. B 65165301

[11] McLaughlin R, Corchia A, Johnston M B, Chen Q, Ciesla C M, Arnone D D, Jones G A C, Linfield E H, Davies A G and Pepper M 2000 Appl. Phys. Lett. 762038

[12] Corchia A, McLaughlin R, Johnston M B, Whittaker D M, Arnone D D, Linfield E H, Davies A G and Pepper M 2001 Phys. Rev. B 64205204

[13] Weiss C, Wallenstein R and Beigang R 2000 Appl. Phys. Lett. 774160

[14] Johnston M B, Whittaker D M, Dowd A, Davies A G, Linfield E H, Li X and Ritchie D A 2002 Opt. Lett. 271935 